\title{
Otorhinolaryngology and Artificial Intelligence
}

\author{
Editorial \\ Turgut Karlıdağ (i) \\ Department of Otorhinolaryngology, Fırat University School of Medicine, Elazığ, Turkey
}

ORCID ID of the author: T.K. 0000-0003-2748-7309.

Cite this article as: Karlıdağ T. Otorhinolaryngology and Artificial Intelligence. Turk Arch Otorhinolaryngol 2019; 57(2):59-60.

\section{Corresponding Author:} Turgut Karlıdağ; turgut_karlidag@yahoo.com DOl: 10.5152/ta0.2019.36116

\section{(c) (1)}

Content of this journal is licensed under a Creative Commons Attribution 4.0 International License.

Available online at www.turkarchotolaryngol.net
Artificial intelligence (AI) is a set of data-oriented techniques and algorithms that are arranged to make predictions or deductions from new data in light of past data. The term AI was coined by John McCarthy as "the science and engineering of developing intelligent computer programs". Since AI is also a collection of various technologies aimed at automating human intellectual processes, different AI technologies are designed for different practices (1). These technologies are reckoned to significantly reduce the need for human labor power in many fields in the near future.

Artificial intelligence is seen to increasingly remain at the forefront of academic research and popular culture discussions. A search was conducted in the PubMed database in June 2019 for the term "artificial intelligence" in order to identify the usage of AI techniques in the medical literature and this search returned 88,727 articles. The number of articles on AI were seen to have increased almost twofold from the previous year.

The application of AI techniques in medicine is relatively new. The purpose of AI in medicine is not to replace the physician, but rather to provide them with advice and recommendations based on the patient data. In the near future, especially the evaluation of the surgical anatomy and anomalies in radiological sections, fast analysis of intraoperative surgical specimens or fine needle aspiration biopsy samples will be possible with the help of AI algorithms $(1,2)$.
While the use of AI seems to be a revolutionary event in the provision of healthcare services, today, its use in the field of otorhinolaryngology, both in practice and in patient care, is limited. Otorhinolaryngologists are key stakeholders in the development and clinical integration of AI technologies. Therefore, collecting high-quality data about patients and diseases is essential for the advancement of AI technologies (3). The first examples of machine learning, a subset of AI, in otorhinolaryngology are used for the automatic recognition of auditory brainstem response waveforms, and the classification of acoustic sound features $(4,5)$.

Malignancies of the head and neck are the most common cases in which AI technologies are used in otorhinolaryngology. AI has been used alongside hyperspectral imaging for differentiating between normal head and neck tissue and thyroid malignancies, and is reported to function with $97 \%$ sensitivity, $96 \%$ specificity, and $96 \%$ accuracy. The system is said to enable surgeons to more precisely define resection margins during procedures (6). Moreover, clinicopathological and genomic markers have been integrated into this system, and successful results have been obtained in predicting the prognosis of cancer patients. Another promising application of $\mathrm{AI}$ is automatic radiotherapy planning. Three-dimensional tumor volumes, especially, can be computed and dosing can be planned more realistically.

Similarly, AI technologies are used in determining the prognosis of sudden sensorineural hearing 
losses in otology, in the imaging of endolymphatic hydrops, and in identifying the phenotypes of hearing loss (7). In laryngology, T1a glottic cancers and other pathologies of the vocal cord can be diagnosed with almost 100\% accuracy when AI programs are used together with voice analysis and videostroboscopy images (8). While the use of AI in rhinology is limited, studies in paranasal sinuses, diagnosis of sinusitis and detection of sinus pathologies are continuing (9).

Although AI applications stand to reduce the need for physicians in the laboratory branches of medicine in the near future, this development may not be as rapid in the clinical and the surgical branches. Nevertheless, it is clear that using AI will provide substantial guidance to physicians in challenging decisions, complex diagnoses and treatments.

\section{References}

1. Crowson MG, Ranisau J, Eskander A, Babier A, Xu B, Kahmke $\mathrm{RR}$, et al. Contemporary review of machine learning in otolaryngology-head and neck surgery. Laryngoscope. 2019 Feb 1. doi: 10.1002/lary.27850. [CrossRef]

2. Janowczyk A, Madabhushi A. Deep learning for digital pathology image analysis: a comprehensive tutorial with selected use cases. J Pathol Inform 2016; 7: 29. [CrossRef]
3 Bur AM, Shew M, New J Artificial intelligence for the otolaryngologist: A state of the art review. Otolaryngol Head Neck Surg 2019; 160: 603-11. [CrossRef]

4. Freeman DT. Computer applications in otolaryngology: computer recognition of brain stem auditory evoked potential wave $\mathrm{V}$ by a neural network. Ann Otol Rhinol Laryngol 1992; 101: 782-90. [CrossRef]

5. Schönweiler R, Hess M, Wübbelt P, Ptok M. Novel approach to acoustical voice analysis using artificial neural networks. J Assoc Res Otolaryngol 2000; 1: 270-82.

6. Halicek M, Lu G, Little JV, Wang X, Patel M, Griffith CC, et al. Deep convolutional neural networks for classifying head and neck cancer using hyperspectral imaging. J Biomed Opt 2017; 22: 60503. [CrossRef]

7. Liu GS, Zhu MH, Kim J, Raphael P, Applegate BE, Oghalai JS. ELHnet: a convolutional neural network for classifying cochlear endolymphatic hydrops imaged with optical coherence tomography. Biomed Opt Express 2017; 8: 4579-94. [CrossRef]

8. Unger J, Lohscheller J, Reiter M, Eder K, Betz CS, Schuster M. A noninvasive procedure for early-stage discrimination of malignant and precancerous vocal fold lesions based on laryngeal dynamics analysis. Cancer Res 2015; 75: 31-9. [CrossRef]

9. Chowdhury NI, Smith TL, Chandra RK, Turner JH. Automated classification of osteomeatal complex inflammation on computed tomography using convolutional neural networks. Int Forum Allergy Rhinol 2019; 9: 46-52. [CrossRef] 